\title{
Neuromuscular junction voice disorders. proposal of a diagnostic protocol
}

\begin{abstract}
Objective: To suggest a diagnostic protocol that evaluates voice alterations as a primary symptom of neuromuscular junction disorders (NMJD).

Data sources: Medline, Web of Science, and Cochrane Library search of the literature from 1979 to 2013

Review methods: Review of literature and data analysis for patients examined in the Puerta de Hierro-Majadahonda University Hospital Voice Clinic from January 2010 to September 2013.

Results: We present our diagnostic protocol when a neuromuscular condition affecting the voice is suspected, emphasizing its benefits in NMJD. Neurophysiological studies are essential in the diagnosis, and we propose to perform them initially. Since NMJD could be due to secondary causes, we begin with electroneurography (ENG) to rule out neuropathies or motor neuron diseases. We follow with electromyography (EMG) to rule out myopathies that could also give secondary NMJD. If ENG and EMG were normal, repetitive nerve stimulation (RNS) is performed. Incremental or decremental responses would indicate different primary NMJD. If RNS was normal, but there is a high suspicion of NMJD, single fiber electromyography (SFEMG) is done. According to these results, laboratory tests are added. Tensilon and acetylcholine receptor antibody (AChR Ab) tests are equivocal and their role is discussed as part of the protocol.

Conclusion: A detailed neurolaryngological examination along with neurophysiological studies can help otolaryngologists diagnose neuromuscular disorders when the altered voice is the primary complaint. Partnership with neurophysiologists and neurologists greatly improves patient care.
\end{abstract}

Keywords: myasthenia, neurolaryngology, electromyography, repetitive nerve stimulation, neuromuscular junction, electroneurography
Volume 5 Issue I - 2016

\author{
Górriz Gil C,' Martínez Pérez F,' ${ }^{2}$ illarreal \\ Patiño IM, ${ }^{3}$ Álvarez Montero O,' Rodriguez \\ Valiente A,' Pinilla Urraca M,' García \\ Berrocal JR' \\ 'Department of Otorhinolaryngology, Puerta de Hierro- \\ Majadahonda University Hospital, Autonomous University of \\ Madrid, Spain \\ 2Department of Clinical Neurophysiology, Puerta de Hierro- \\ Majadahonda University Hospital,Autonomous University of \\ Madrid, Spain \\ ${ }^{3}$ Department of Otorhinolaryngology, La Milagrosa Hospital, \\ Autonomous University of Madrid, Spain
}

Correspondence: Carmen Górriz Gil, Department of Otorhinolaryngology Head \& Neck Surgery, C/ Joaquin Rodrigo, 2, 28002 Majadahonda, Madrid, Spain, Tel +34 630955454, Email carmengorriz@yahoo.co.uk

Received: July 09, 2016 | Published: November 07, 2016
Abbrevations: NMJD, neuromuscular junction disorders; ENG, electro neurography; EMG, electro myography; RNS, repetitive nerve stimulation; SFEMG, single fiber electromyography; AChR Ab, acetylcholine receptor antibody; anti-MuSk $\mathrm{Ab}$, anti-muscle specific kinase antibody; TA, tyro arythenoid muscle; CMAP, compound motor action potential; Lrp4 Ab, lipoprotein receptor-related protein 4 antibody; CT, computed tomography; Lrp4, lipoprotein receptorrelated protein 4

\section{Introduction}

The larynx and pharynx neuromuscular balance has to be intact to be able to fine tune swallowing, breathing and voice production. Dysphonia could be the presenting symptom of a general neuromuscular condition and the otolaryngologist the first specialist to be consulted. Knowledge and awareness of the neuromuscular diseases, the signs and symptoms that could impair the voice and swallowing, along with familiarity performing a detailed neurological pharyngolaryngeal examination is essential to diagnose neurological diseases long before they are more widespread and obvious. ${ }^{1-3}$ If dysphagia, lack of fluency or altered prosody is present, a neuromuscular origin should be suspected (Table 1).

Neuromuscular junction disorders rarely affect the voice as a primary symptom. ${ }^{3-}$ In the literature they are classified into primary and secondary conditions. Myasthenia is the most common NMJD. Fluctuating vocal muscle weakness, dysarthria and dysphagia are the main symptoms. Neurophysiological studies are critical in the evaluation of the patient with suspected NMJD: nerve conduction studies or ENG, EMG, RNS and SFEMG. RNS is the most important test in detecting NMJD but it is not specific for these conditions. ${ }^{6}$ Serologic testing of antiACh-R Ab, and anti-muscle specific kinase antibody (anti-MuSk Ab) establishes a correct diagnosis but they tend to be negative in laryngeal or localized forms. Anticholinesterase test helps to confirm diagnosis but it is not specific. ${ }^{7-9}$

We present our diagnostic protocol when neuromuscular conditions, due to voice abnormalities as the main symptom, are suspected, with special emphasis in NMJD.

\section{Material and methods}

Our routine neurolaryngological examination protocol is as follows:

\section{Medical history}

Since neuromuscular conditions affect swallowing, breathing and voice production, a questionnaire about the three systems should be routinely made. ${ }^{10}$ The symptoms that could elicit a neuromuscular condition are not very specific (Table 1). Searching for drugs that could affect neuromuscular physiology such as statins, botulin toxin, certain antibiotics, antacids and laxatives containing magnesium is mandatory. ${ }^{10-12}$ Any recently passed viral infection should be taken into account. 
Table I Signs and symptoms of neuromuscular disorders. Modified of Rubin'

\begin{tabular}{|c|c|c|c|}
\hline Site of Lesion & Laryngeal Findings & Vocal/ Swallowing Symptoms & Neurologic Diseases \\
\hline $\begin{array}{l}\text { Upper Motor Neuron } \\
\text { (bilateral) }\end{array}$ & $\begin{array}{l}\text { Spastic vocal fold paresis/ } \\
\text { paralysis }\end{array}$ & $\begin{array}{l}\text { Strained voice, breathy voice, } \\
\text { laryngospasm, breathing, } \\
\text { dysarthria incoordination, } \\
\text { altered prosody }\end{array}$ & $\begin{array}{l}\text { Amyotrophic lateral sclerosis, Progressive } \\
\text { lateral sclerosis, Pesudobulbar palsy }\end{array}$ \\
\hline Lower Motor Neuron & $\begin{array}{l}\text { Flaccid vocal paresis/paralysis, } \\
\text { glottic insufficiency }\end{array}$ & $\begin{array}{l}\text { Weak, breathy voice, } \\
\text { hypernasal speech, } f \\
\text { laccid dysarthria, } \\
\text { nasal regurgitation, } \\
\text { oropharyngeal dysphagia, } \\
\text { altered prosody? }\end{array}$ & $\begin{array}{l}\text { Amyotrophic lateral sclerosis, Progressive } \\
\text { bulbar palsy, Spinal muscle atrophy }\end{array}$ \\
\hline Extrapyramidal & $\begin{array}{l}\text { Vocal fold bowing, tremor, } \\
\text { dystonia, dysdiadochokinesia }\end{array}$ & $\begin{array}{l}\text { Tremor, laryngeal dystonia, } \\
\text { weak, breathy voice, monotone, } \\
\text { dysphagia }\end{array}$ & $\begin{array}{l}\text { Parkinson, Spasmodic dysphonia, } \\
\text { laryngeal tremor }\end{array}$ \\
\hline Peripheral Nerve & Paresis/paralysis & $\begin{array}{l}\text { Weak, breathy voice, } \\
\text { hypernasal speech, } \\
\text { ysphagia, aspirations, } \\
\text { pitch abnormalities }\end{array}$ & Vagal nerve, SLN, RLN injury \\
\hline Myopathy & Paresis, flaccidity & Weak, breathy voice & Myopathies \\
\hline $\begin{array}{l}\text { Neuromuscular Junction } \\
\text { Disorder }\end{array}$ & Fluctuating paresis/paralysis & $\begin{array}{l}\text { Weak, breathy voice, dysarthria, } \\
\text { dysphagia, vocal fatigue, altered } \\
\text { resonance }\end{array}$ & Myasthenia \\
\hline
\end{tabular}

The patient with laryngeal NMJD could complain of dysphagia, dysarthria, hypernasality, nasal regurgitation or problems with vocal articulation. Shortness of breath could be a sign of chest muscle weakness that would worsen their vocal function. Patients with myasthenia would generally feel more tired than normal even when chewing. Symptoms tend to be asymmetric in distribution and fluctuating in intensity. ${ }^{4,5,13}$

\section{Physical examination}

Physical exam usually begins with observation of the general appearance of the patient and attentive listening to the voice. Abnormal facial expression ${ }^{1,14}$ or posturing, tremor, tone deficiency, lack of fluency, articulation problems, spastic voice breaks or altered prosody should be noted. Seek for signs of low cranial nerve impairment such as palatal muscle weakness, tongue movement impairment or fasciculation.

The psychoacoustic analysis would involve assessing the voice with different tasks: reading a text at conversational and projected voice, forceful yell and performing "glissandos". The GRABS scale is noted. Fluidity, pitch control and articulation are observed. In NMJD breathiness and asthenia are frequently present with variable degree and fluctuating pattern during the day. Strained vocal quality could be present if compensatory muscle effort exists. Maximum phonation time is decreased and the phonorespiratory index could be normal due to lower respiratory function in myasthenia patients. When this finding is encountered we have to make the differential diagnosis with restrictive pulmonary diseases or with other neuromuscular myopathies.

Flexible laryngoscopy allows physiological laryngeal behaviour and assesses larynx symmetry from nasopharynx to glottis; any rotation could elicit nerve paresis or muscular compensation. The vocal fold itself tends to be normal on fibroendoscopic examination, but in the majority of patients an incomplete glottic closure is encountered leading to a misdiagnosis of vocal fold atrophy or even the presence of a sulcus.
Spontaneous movements at rest must be observed. Vocal fold mobility should be assessed with different tasks: spontaneous and projected speech, whistling, singing and glissandos. Fatigability and agility can be noticed by alternating forced breathing and a vocal sound (sniff-/i/) as well as the sound /i/-/hi/ repetitively. Check coordination with alternating abduction and adduction movements with sounds such as $/ \mathrm{pa} /-/ \mathrm{ta} /-/ \mathrm{ka} /$ respectively. ${ }^{15}$ Note muscle tone and tyroarythenoid (TA) bulk as well as any hyper functional or hypo functional behaviours.

Differentiate from psychological conditions asking to perform tricky vocal functions such as coughing, or inverse phonation. If an ample hiatus is present and malingering is suspected make the patient phonate /e/ while holding his tongue to disrupt an abnormal learnt behaviour. We recommend being as close as possible to the vocal folds to check for fasciculations, muscle bulk or tension abnormalities and size of the ventricles. This gives an insight of reinnervation in the TA muscle. If gag reflex is prominent we can suppress it with $4 \%$ lidocaine, administered either through the channel fiberscope or injected in the cricothyroid membrane. Stroboscopic examination with flexible endoscope should be performed to elicit any lesions that could impair mucosal wave. Assessment of mucosal symmetry, height of the vocal processes, amplitude of mucosal wave and muscle tonicity is observed. Any asymmetry of the vocal fold mucosal wave, in absence of organic lesion, could elicit different muscle tension of the TA.

Once laryngeal NMJD is suspected based on clinical findings, the diagnosis should be confirmed on clinical, neurophysiological and serological tests.

\section{Neurophysiological studies (Figure I)}

RNS has been refined and validated as one of the most useful neurophysiological tests in the evaluation of patients with suspected primary NMJD. It is abnormal in $76 \%$ of patients with generalized myasthenia but its sensitivity drops to $48 \%$ in patients with the restricted form of myasthenia. ${ }^{16-18}$ A decremental response on RNS 
is the electrical correlation of clinical muscle fatigue and weakness, and can be seen in various neurogenic and myogenic conditions (e.g., neuropathies, myopathies, motor neuron disorders), in addition to primary NMJD.

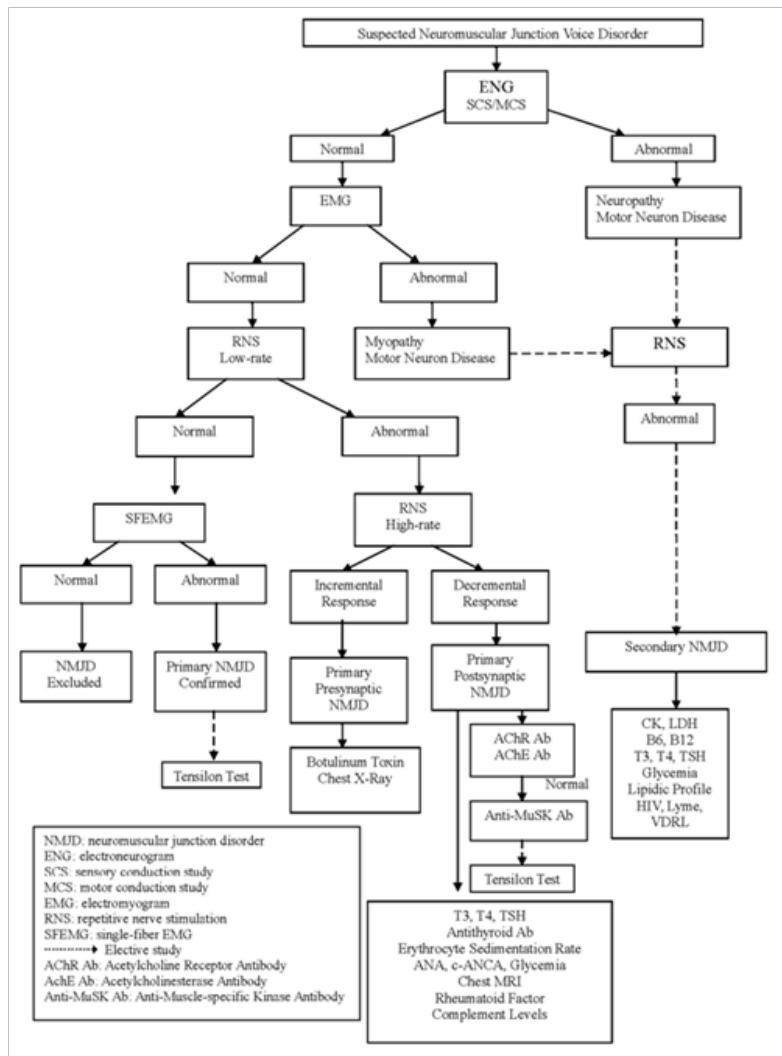

Figure I Diagnostic protocol in suspected neuromuscular junction voice disorders.

For this reason, our protocol starts with an ENG study. One sensory and one motor nerve in upper and lower extremities are examined unilaterally for screening of a peripheral neuropathy or motor neuron disease. The compound motor action potential (CMAP) should be normal in patients with NMJD. Only a small number of patients have a baseline CMAP that falls below the normal range. If low amplitude CMAPs is registered, Lambert-Eaton myasthenic syndrome should be considered.

If the ENG is normal and since the neuromuscular junction can be secondarily affected by myopathies or motor neuron disease, an EMG should be scheduled. We evaluate a proximal and a distal muscle both in upper and lower extremities unilaterally in order to rule out generalized disorders, followed by a laryngeal electromyography. ${ }^{19,20}$

Once integrity of nerve and muscle has been confirmed, RNS should be scheduled. At least one distal and one proximal nerve are recommended. Low frequency $(3 \mathrm{~Hz})$ RNS should be first performed $^{16,17}$ in a distal nerve (ulnar nerve-abductor digiti minimi muscle). Despite lower sensitivity, it is better tolerated and less prone to technical artifacts. If normal, then a proximal nerve should be used (spinal accessory nerve-superior trapezius muscle). If both are normal, superior laryngeal nerve-cricothyroideus muscle should be considered. A decrement of $10 \%$ on slow frequency RNS is characteristically seen in patients with NMJD. ${ }^{21}$

After low frequency RNS, high frequency $(20 \mathrm{~Hz})$ or postexercise testing is performed. Incremental responses are suggestive of presynaptic NMJD. Decremental responses of more than $10 \%$ and blocking are suggestive of postsynaptic NMJD. In patients with normal RNS but a high clinical suspicion of NMJD, a SFEMG is performed looking for increased jitter or blocks.

Voluntarily activated SFEMG ${ }^{16}$ is used to measure the relative firing of adjacent single muscle fibers from the same motor unit and can detect both prolonged jitter-the measurement of the mean value of consecutive differences of successive inter potential intervals-, as well as blocking of muscle fibers (when impulses intermittently fail to occur). SFEMG is the most sensitive test for detecting abnormalities consistent with myasthenia, ${ }^{18}$ up to $97 \%$ in ocular myasthenia, and $99 \%$ in generalized myasthenia when appropriate muscles are sampled. However, its usefulness is compromised by its time-intensiveness and requirement for specialized equipment and training. Therefore, it should be used only by experienced clinical neurophysiologists in the definitive diagnosis of suspected myasthenic patients, whose other diagnostic tests are normal and who do not harbor other neuromuscular diseases. $^{21}$

\section{Laboratory and radiology tests}

These tests are performed according to the clinical and neurophysiological results. We propose a sequential fashion. If the ENG is abnormal, as neuropathy or motor neuron disease is suspected, tests to investigate the cause should be started (Figure 1). If ENG is normal, but EMG is abnormal, myopathy tests should be added.

In case a presynaptic NMJD is suspected, botulinum toxin test and chest RX should be performed. If decremental response were to be found, primary postsynaptic NMJD should be suspected and AChR $\mathrm{Ab}$ and acetylcholinesterase antibody (AChE $\mathrm{Ab}$ ) added. These antibodies tend to be positive in generalized myasthenia but are less likely to have abnormal results in the laryngeal form. In patients who are negative for AChR antibodies, test for MuSK antibodies and the newly identified lipoprotein receptor-related protein 4 antibody (Lrp4 $\mathrm{Ab}$ ) should be performed. ${ }^{22}$

Tensilon test (intravenous injection of the acetylcholinesterase inhibitor, edrophonium chloride) is not specific for myasthenia, but it could support the diagnosis along with the neurophysiological studies. $^{23}$ The subjective response considered positive is an improvement in the quality of voice because of the immediate increase in muscle strength. ${ }^{24}$ The objective response will be the increase in the maximum phonation time of a vowel. The maximum expiratory time tends to increase as well if respiratory muscles are involved.

Edrophonium is given in a gradually incremental dose starting with $2 \mathrm{mg}$ intravenously. If after 2 minutes no clear response develops, up to 8 additional $\mathrm{mg}$ could be injected. The response will last no longer than 5 minutes. The test could be started with a placebo of $2 \mathrm{ml}$ of saline. Care must be taken for cholinergic effects, such as bradycardia and atropine has to be available in case they occur, administering up to $1 \mathrm{mg}$ intravenously or subcutaneously.

An evaluation of commonly associated diseases is required in every myasthenia case. Thyroid dysfunction is a frequent accompaniment and may aggravate myasthenic weakness as well as rheumatoid arthritis and systemic lupus erythematosus. Computed tomography (CT) or magnetic resonance of the chest is needed to evaluate for thymoma or other thymic abnormalities. ${ }^{25-27}$

\section{Discussion}

Very often otolaryngologists are the initial specialists to be consulted for dysphonia that could elicit the first and only sign of 


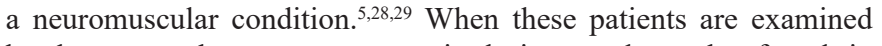
by laryngostroboscopy no organic lesion tends to be found in the vocal folds. The otolaryngologist has to be familiar with the neurolaryngologic conditions that could affect the voice and be able to routinely perform a protocolled neurolaryngologic examination of voice, swallowing and breathing. ${ }^{2,30,31}$ We have presented our protocol with emphasis on electrophysiological studies and NMJD.

Our medical history and physical examination follows and confirms the advice of other authors. ${ }^{1,2,14}$ The effect of drugs such as statins and certain antibiotics could mimic neuromuscular conditions. ${ }^{11}$ Very suggestive of NMJD are: change in vocal volume or resonance, vocal fatigability, altered articulation and swallowing or chewing problems that typically fluctuate with time. Mild general fatigue tends to be present. The patient may not refer initially all the symptoms. ${ }^{4,32,33}$

On physical examination abnormal facial expression, general appearance of tiredness, hyper nasality and articulation problems are typical of NMJD due to muscles weakness. The voice is of low volume and at best is high pitched due to crycothyroid muscle compensation. Phonorespiratory index is important to elicit respiratory muscle weakness or other restrictive pulmonary conditions. Fluctuation of signs and fatigue in repeated tasks are likely to be caused by NMJD. Asymmetric vocal fold mass is common in these cases whose vocal folds would move with different frequency showing an asymmetric vocal fold movement and a bitonal voice. Close contact to the vocal fold is necessary to view any fasciculations that could elicit synkinesis or myopathies and to assess the bulk of the TA.

The diagnosis should be confirmed first with neurophysiological studies according to our protocol. Since a decrement on RNS can be seen in various neurogenic and myogenic conditions in addition to primary NMJD, the first test we recommend to perform is an ENG of a sensory and of a motor nerve, to be sure that the nerves tested in the RNS are normal. If ENG is normal, EMG is performed to rule out neuromyopathies. Only with normal ENG and EMG, a primary NMJD can be diagnosed. RNS is moderately sensitive, detecting only $68 \%$ of $\mathrm{NMJD}^{33}$ and in laryngeal forms only from 15 to $36 \%{ }^{5,6}$ This could be avoided testing proximally if distally is negative and suspicion is high as sensitivity increases from distal to proximal muscles when performed on clinically weak muscles. CT muscle RNS would be the last proximal muscle to be tested. Testing the TA would be useful but it is very painful, prone to spasmodic reaction or rupture and potentially dangerous, although some authors are in favor. ${ }^{33}$ They find positive RNS in laryngeal muscles in $87 \%$ of myasthenia patients with laryngeal complaints being the CT muscle the most frequently involved in $93 \%$ cases, compared to $50 \%$ in TA muscles. These high rates could be due to the generalized myasthenia they suffered from and not only from laryngeal involvement. That could be avoided by performing SFEMG. Again, SFEMG without ENG would give many false positive, primary NMJD, as it would be abnormal in most neuromyopathies. Contrarily, if SFEMG is normal, primary NMJD can be excluded. Another problem of SFEMG is that it does not differentiate between primary and secondary NMJD. ${ }^{34}$

When screening a patient for myasthenia, the quantification of $\mathrm{AChR} \mathrm{Ab}$ is the most sensitive test. Between $80-85 \%$ of patients with generalized myasthenia will have elevated binding antibodies, while at best $55 \%$ of ocular myasthenia will be positive. ${ }^{35}$ Nevertheless, seronegativity does not rule out the disease, like in laryngeal myasthenia. ${ }^{4}$ Low seropositivity is postulated to be due to the small amount of circulating antibodies, as laryngeal muscles are very small, generally activated and easily tired, and the antibodies could be bound to the motor end plate. Alternatively the antigen could be different in generalized forms or be a different immune mechanism. ${ }^{4}$
Anti-MuSk Ab are present in up to 38-50 percent of those AChR $\mathrm{Ab}$ negative, generalized myasthenia. ${ }^{36}$ Lipoprotein receptor-related protein 4 (Lrp4) is a newly identified receptor for agrin, essential for activation of MuSK during the formation of the neuromuscular junction. In a small number of patients who are negative for AChR antibodies, IgG1 antibodies against Lrp4 have been described. Although serological testing is a useful adjunct to the diagnosis of myasthenia, a large minority of patients will be seronegative, and confirmation of the diagnosis will rely on other diagnostic testing.

We prefer to use edrophonium (Tensilon) to neostigmine or pyridostigmine (Mestinon) because of its fast onset, acting in 30 seconds in comparison to 20 minutes with neostigmine or 30 minutes with pyridostigmine. Nevertheless its duration is only 5minutes; so vocal tests have to be done quickly. Other authors prefer neostigmine whose duration is 50-60minutes. ${ }^{5}$ We consider the maximum phonation time a very good objective indicator of vocal strength improvement. We find EMG changes difficult to evaluate and less objective in comparison with other authors that used them. ${ }^{4}$ Improvement in RNS is another objective measure but in our experience the responses are not consistent enough. Maximum expiratory time tends to be impaired and its improvement benefits voice production. Pitch control when singing and increase in fundamental frequency are also good improvement indicators. ${ }^{4}$ Any of the above should be considered positive responses in our opinion. Tensilon test cannot be performed initially as many false positive, primary NMJD will be diagnosed. Most secondary NMJD will also improve with Mestinon, as the end plate is secondarily affected. Furthermore, although Tensilon test is positive in $95 \%$ of generalized myasthenia it is less sensitive in laryngeal forms with $50 \%$ false negative rate. ${ }^{37}$ The possibility of hypothyroidism and myasthenia together is not rare, since they are both of autoimmune origin with 5 to $23 \%$ adjunction of autoimmune conditions reported. ${ }^{26}$

\section{Conclusion}

Voice complaints could be the first sign of neuromuscular disorders and the otolaryngologist has to be aware of this and should routinely perform a neurolaryngologic examination. Neurophysiological studies (ENG, EMG, RNS and SFEMG) are essential to come to a diagnosis and should be performed before laboratory studies. Initial RNS or Tensilon test could lead to false positive, primary NMJD since neuromyopathies could affect the end plate secondarily. $\mathrm{AChR}-\mathrm{Ab}$ and Anti-MuSk Ab, are generally negative in laryngeal myasthenia, although if they were positive, they would support the diagnosis. We propose a protocol with ENG as a first step followed by EMG and RNS. ${ }^{38}$ Laboratory tests will be performed according to the diagnostic protocol. If primary myasthenia is diagnosed, other autoimmune disorders should be ruled out. Multidisciplinary work with neurophysiologist and neurologists greatly improves diagnosis and patient care.

\section{Classification of neuromuscular junction disorders}

\section{Primary}

A. Presynaptic

a. Lambert-Eaton

b. Botulism

c. Magnesium-induced paralysis

B. Postsynaptic 
a. Myasthenia

b. Myasthenic syndromes

C. Acetyl cholinesterase deficit

a. Drug induced: organophosphate and curare poisoning

b. Channelopathies

D. Combined

a. Drug induced: aminoglycoside, quinine, statin, $\mathrm{Mg}++$ drugs, botulin toxin, Calcium channel blockers

b. Congenital myasthenic syndromes

\section{Secondary}

\section{A. Central}

a. Supranuclear (first motor neuron)

b. Nuclear or bulbar (second motor neuron)

B. Peripheral

a. Peripheral neuropathy

b. Myopathy

C. Inflammatory

D. Non inflammatory

\section{Acknowledgments}

None.

\section{Conflicts of interest}

Author declares there are no conflicts of interest.

\section{Funding}

None.

\section{References}

1. Rubin AD. Neurolaryngologic evaluation of the performer. Otolaryngol Clin North Am. 2007;40(5):971-989.

2. Woodson G. Management of neurologic disorders of the larynx. Ann Otol Rhinol Laryngol. 2008;117(5):317-326.

3. Merati AL. In-office evaluation of swallowing: FEES, pharyngeal squeeze maneuver, and FEESST. Otolaryngol Clin North Am. 2013;46(1):31-39.

4. Mao VH, Abaza M, Spiegel JR, et al. Laryngeal myasthenia gravis: report of 40 cases. $J$ Voice. 2001;15(1):122-130.

5. Liu WB, Xia Q, Men LN, et al. Dysphonia as a primary manifestation in myasthenia gravis (MG): a retrospective review of 7 cases among 1520 MG patients. J Neurol Sci. 2007;260(1-2):16-22.

6. Naeije G, Legros B, de Beyl DZ. Neuromuscular junction border conflict. Journal of Neurology. 2010;257(8):1402-1403.

7. Ohta K, Fujinami A, Saida T, et al. Frequency of anti-AChR epsilon subunit-specific antibodies in MG. Autoimmunity. 2003;36(3):151-154.

8. Leite MI, Jacob S, Viegas S, et al. IgG1 antibodies to acetylcholine receptors in "seronegative" myasthenia gravis. Brain. 2008;131(Pt 7):1940-1952.

9. Jacob S, Viegas S, Leite MI, et al. Presence and pathogenic relevance of antibodies to clustered acetylcholine receptor in ocular and generalized myasthenia gravis. Arch Neuro. 2012;69(8):994-1001.
10. Castelbaum AR, Donofrio PD, Walker FO, et al. Laxative abuse causing hypermagnesemia, quadriparesis, and neuromuscular junction defect. Neurology. 1989;39(5):746-747.

11. Parmar B, Francis PJ, Ragge NK. Statins, fibrates, and ocular myasthenia. Lancet. 2002;360(9334):717.

12. Iwase T, Iwase C. Systemic effect of local and small-dose botulinum toxin injection to unmask subclinical myasthenia gravis. Graefes Arch Clin Exp Ophthalmol. 2006;244(3):415-416.

13. Carpenter RJ, McDonald TJ, Howard FM. The otolaryngologic presentation of myasthenia gravis. The Laryngoscope. 1979;89(6 Pt 1):922-928.

14. Richardson BE, Bastian RW. Clinical evaluation of vocal fold paralysis. Otolaryngol Clin North Am. 2004;37(1):45-58.

15. Heman-Ackah YD, Barr A. Mild vocal fold paresis: understanding clinical presentation and electromyographic findings. $J$ Voice. 2006;20(2):269-281.

16. AAEM Quality Assurance Committee, American Association of Electrodiagnostic Medicine. Practice parameter for repetitive nerve stimulation and single fiber EMG evaluation of adults with suspected myasthenia gravis or Lambert-Eaton myasthenic syndrome: summary statement. Muscle Nerve. 2001;24(9):1236-1238.

17. Chiou-Tan FY. Electromyographic approach to neuromuscular junction disorders repetitive nerve stimulation and single-fiber electromyography. Phys Med Rehabil Clin N Am. 2003;14(2):387-401.

18. Sanders DB. Electrophysiologic tests of neuromuscular transmission. Supplements to Clinical Neurophysiology. 2004;57:167-169.

19. Volk GF, Hagen R, Pototschnig C, et al. Laryngeal electromyography: a proposal for guidelines of the European Laryngological Society. Eur Arch Otorhinolaryngol. 2012;269(10):2227-2245.

20. Blitzer A, Crumley RL, Dailey SH, et al. Recommendations of the Neurolaryngology Study Group on laryngeal electromyography. Otolaryngology-Head and Neck Surgery. 2009;140(6):782-793.

21. Katirji B, Kaminski HJ, Ruff RL. Neuromuscular Disorders in Clinical Practice. (2nd edn), Springer. 2013. p.1565.

22. Pevzner A, Schoser B, Peters K, et al. Anti-LRP4 autoantibodies in AChR-and MuSK-antibody-negative myasthenia gravis. $J$ Neurol. 2012;259(3):427-435.

23. Daroff RB, Mazziotta JC. Neurology in Clinical Practice. (6th edn), Saunders, USA. 2012. p. 2544.

24. Sataloff RT, Mandel S, Heman-Ackah YD, et al. Laryngeal Electromyography. (2nd edn), Plural Publishing, San Diego, USA. 2005. p. $47-101$.

25. Mamarabadi M, Razjouyan H, Moghaddasi M. Hypothyroidism, the main thyroid dysfunction in Iranian patients with myasthenia gravis: A case serie. Iran J Neurol.2011;10(1-2):22-25.

26. Cavalcante P, Le Panse R, Berrih-Aknin S, et al. The thymus in myasthenia gravis: Site of "innate autoimmunity"? Muscle Nerve. 2011;44(4):467-484.

27. Heman-Ackah YD, Joglekar SS, Caroline M, et al. The prevalence of undiagnosed thyroid disease in patients with symptomatic vocal fold paresis. J Voice. 2011;25(4):496-500.

28. San Martín GG, Górriz C, Martín L, et al. Dysphonia as a first symptom in several neuromuscular diseases. Laryngeal electroneuromyography diagnostic utility. Neurologia. 2009;24(9):819-821.

29. Cucurachi L, Cattaneo L, Gemignani F, et al. Late onset generalized myasthenia gravis presenting with facial weakness and bulbar signs without extraocular muscle involvement. Neurological Sciences. 2009;30(4):343-344. 
30. Gamboa J, Jiménez-Jiménez FJ, Mate MA, et al. Voice disorders caused by neurological diseases. Rev Neurol. 2001;33(2):153-168.

31. Merati AL, Heman-Ackah YD, Abaza M, et al. Common movement disorders affecting the larynx: a report from the neurolaryngology committee of the AAO-HNS. Otolaryngol Head Neck Surg. 2005;133(5):654-665.

32. Salazar C, De Saa MR, Aparicio M, et al. Myasthenia gravis Otorhinolaryngological considerations. Acta Otorrinolaringol Esp. 2000;51(1):92-96.

33. Xu W, Han D, Hou L, et al. Clinical and electrophysiological characteristics of larynx in myasthenia gravis. Ann Otol Rhinol Laryngol. 2009;118(9):656-661.

34. Howard JF. Electrodiagnosis of disorders of neuromuscular transmission. Phys Med Rehabil Clin N Am. 2013;24(1):169-192.
35. Garlepp MJ, Dawkins RL, Christiansen FT, et al. Autoimmunity in ocular and generalised myasthenia gravis. $J$ Neuroimmunol. 1981;1(3):325-332.

36. Suzuki S, Utsugisawa K, Nagane Y, et al. Classification of myasthenia gravis based on autoantibody status. Arch Neurol. 2007;64(8):1121-1124.

37. Higuchi O, Hamuro J, Motomura M, et al. Autoantibodies to low-density lipoprotein receptor-related protein 4 in myasthenia gravis. Ann Neurol. 2011;69(2): 418-422.

38. Heitmiller RF. Myasthenia gravis: clinical features, pathogenesis, evaluation, and medical management. Semin Thorac Cardiovasc Surg. 1999;11(1):41-46. 